Article

\title{
An Efficient Application of the MOEA/D Algorithm for Designing Noise Abatement Departure Trajectories
}

\author{
Vinh Ho-Huu ${ }^{\dagger}$, Sander Hartjes ${ }^{*}{ }^{\dagger}$, Hendrikus G. Visser (D) and Richard Curran \\ Faculty of Aerospace Engineering, Delft University of Technology, 2629 HS Delft, The Netherlands; \\ v.hohuu@tudelft.nl (V.H.-H.); h.g.visser@tudelft.nl (H.G.V.); r.curran@tudelft.nl (R.C.) \\ * Correspondence: s.hartjes@tudelft.nl; Tel.: +31-15-27-88925 \\ + These authors contributed equally to this work.
}

Received: 2 October 2017; Accepted: 27 October 2017; Published: 1 November 2017

\begin{abstract}
In an effort to allow to increase the number of aircraft and airport operations while mitigating their negative impacts (e.g., noise and pollutant emission) on near-airport communities, the optimal design of new departure routes with less noise and fuel consumption becomes more important. In this paper, a multi-objective evolutionary algorithm based on decomposition (MOEA/D), which recently emerged as a potential method for solving multi-objective optimization problems (MOPs), is developed for this kind of problem. First, to minimize aircraft noise for departure routes while taking into account the interests of various stakeholders, bi-objective optimization problems involving noise and fuel consumption are formulated where both the ground track and vertical profile of a departure route are optimized simultaneously. Second, in order to make the design space of vertical profiles feasible during the optimization process, a trajectory parameterization technique recently proposed is employed. Furthermore, some modifications to MOEA/D that are aimed at significantly reducing the computational cost are also introduced. Two different examples of departure routes at Schiphol Airport in the Netherlands are shown to demonstrate the applicability and reliability of the proposed method. The simulation results reveal that the proposed method is an effective and efficient approach for solving this kind of problem.
\end{abstract}

Keywords: departure routes; trajectory optimization; noise abatement; fuel consumption; MOEA/D

\section{Introduction}

Due to the high demand of air transport, the aviation industry is expected to develop rapidly in the coming years [1]. To adapt to this requirement, the increase of aircraft and airport operations is necessary and important. However, the increase in these operations often results in negative impacts on the quality of life of near-airport communities, such as noise and pollutant emissions [2]. As a result, the protest of communities surrounding airports becomes a major restriction that policymakers have to deal with when accomodating additional operations. In order to develop air transport sustainably, it is important to investigate potential solutions for decreasing its adverse influences. In recent years, a series of projects aiming to develop the aviation sector sustainably have been launched by European and national authorities, including CleanSky [3], the Atlantic Interoperability Initiative to Reduce Emission (AIRE) [4], and the Asia and South Pacific Initiative to Reduce Emission (ASPIRE) [5]. Various strategies have been proposed, such as making new policies and standards, developing advanced aircraft technologies and sustainable alternative fuels, and changing aircraft/airport operational procedures [6]. Among them, the change of aircraft/airport operational procedures may be a potential option in the short-term as it can be adapted more quickly and often at less cost as compared to the 
other options [6]. The optimal design of new routes for departures and arrivasls is one of the possible solutions that has been broadly studied in the past few years $[7,8]$.

In order to design optimal environmentally friendly terminal routes, different approaches have been proposed in recent years. Visser and Wijnen $[9,10]$ developed an optimization tool called NOISHHH that combines a noise model, an emissions inventory model, a geographic information system, and a dynamic trajectory optimization algorithm to generate environmentally optimal departure and arrival trajectories. Later, this tool was also adapted to optimize noise abatement terminal routes based on area navigation [11-13]. Prats et al. [14,15] employed a lexicographic optimization technique to deal with aircraft departure trajectories for minimizing noise annoyance. Khardi and Abdallah [16] studied a comparison of direct and indirect methods in solving a system of ordinary differential equations (ODEs) to optimize aircraft flight paths to reduce noise. Recently, Matthes et al. [17] presented a concept for multi-criteria environmental assessment of aircraft trajectories where the mathematical framework for environmental assessment and optimization of aircraft trajectories was developed. Despite being quite efficient in searching optimal trajectories, these techniques belong to the group of gradient-based methods that have certain limitations in solving optimization problems. For example, due to the use of gradient information to search for an optimal solution, these methods are often only suitable for optimization problems whose objective and constraint functions are differentiable and whose decision variables are continuous. Moreover, their solutions are often trapped in local optima if the considered problems are nonlinear and contain more than one local optimal solution. Nevertheless, current optimization problems become more and more complex due to the integration of operational constraints in realistic scenarios. It is therefore quite difficult to construct differentiable optimization problems. Also, these techniques are single-optimization methods, which means that only a single optimal solution is obtained after each time the optimization problem is solved.

By considering the above research gaps, different gradient-free optimization techniques have also been applied. Torres et al. [18] proposed a non-gradient optimizer called multi-objective mesh adaptive direct search (multi-MADS) to synthesize optimal departure trajectories for $\mathrm{NO}_{\mathrm{x}}$-emissions and noise at a single measurement point. Recently, Hartjes and Visser [19] employed an elitist non-dominated sorting genetic algorithm (NSGA-II) combined with a novel trajectory parameterization technique for the optimal design of departure trajectories with environmental criteria. This approach was then also applied by Zhang et al. [20] to optimize departure routes at Manchester Airport. From the obtained results in [18-20], it is clear that the use of non-gradient multi-objective optimization methods is a potential approach for designing new routes. These methods readily overcome the limitations of gradient-based methods in dealing with discontinuous problems and integer or discrete design variables and can find out a set of non-dominated optimal solutions, which helps to present more options to policymakers and authorities. However, one of the major limitations of the methods in this group is their computational cost. Because they feature random searches with multiple design points at the same time, these methods require many evaluations of the objective and constraint functions which are quite time-consuming. These restrictions again have motivated researchers to develop computationally efficient approaches that can balance the expected results and computation cost effectively.

Until now, besides multi-MADS and NSGA-II, there are various multi-objective optimization algorithms available in the literature that may be considered potential candidates for solving these kinds of problems. However, they have not yet been properly investigated. Among them, the multi-objective evolutionary algorithm based on decomposition (MOEA/D) [21] has recently emerged as a powerful method and has received much attention from researchers. According to recent studies [21,22], MOEA/D has been demonstrated to be more efficient than NSGA-II and some other methods regarding both the quality of solutions and the convergence rate, which are promising features for solving large-scale real-world problems. Nonetheless, the application of MOEA/D to real engineering problems is still somewhat limited, especially in the field of aerospace engineering. 
In this paper, MOEA/D is considered for the optimal design of aircraft noise abatement departure routes. As mentioned before, however, MOEA/D is a gradient-free optimization method, and hence its computation cost is still signficant. In order to make the applied algorithm more efficient, a new implementation in the definition of the optimization problem for MOEA/D is introduced that can help to reduce the computational cost significantly. Furthermore, the efficiency of the MOEA/D version in this paper is also considerably enhanced by the integration of some recently developed advantageous features. These include an adaptive replacement strategy [23], a stopping condition criterion [24], and a constraint-handling technique [25]. Also, to reduce redundant evaluations of infeasible solutions derived from operational constraints during different flight phases, the new trajectory parameterization technique recently proposed in [19] is also applied. The robustness and reliability of the proposed method are validated through two numerical examples at Schiphol Airport in The Netherlands.

\section{Aircraft Model and Trajectory Parameterization Technique}

\subsection{Aircraft Model}

To model an aircraft during departure operations, an intermediate point-mass model [19] is used in this paper. The model is based on several assumptions: (1) there is no wind present; (2) the Earth is flat and non-rotating; and (3) the flight is coordinated. In addition, the flight path angle is considered sufficiently small $\left(\gamma<15^{\circ}\right)$. The underlying assumption for the intermediate model is the equilibrium of forces normal to the flight path. The implication of this simplifying assumption is that the aerodynamic drag is slightly underestimated since it is evaluated as if the aircraft performs a quasi-linear flight. With these assumptions, the equations of motion can be stated as follows:

$$
\begin{gathered}
\dot{V}_{\mathrm{TAS}}=g_{0}\left[\frac{(T-D)}{W}-\sin \gamma\right], \\
\dot{s}=V_{\mathrm{TAS}} \cos \gamma, \\
\dot{h}=V_{\mathrm{TAS}} \sin \gamma, \\
\dot{W}=-\dot{m}_{\mathrm{f}} g_{0},
\end{gathered}
$$

where $\dot{V}_{\mathrm{TAS}}, \dot{s}, \dot{h}$, and $\dot{W}$ are the time derivatives of the true airspeed, ground distance flown, altitude, and aircraft weight, respectively; $g_{0}$ is the gravitational acceleration, and $T, D$, and $\dot{m}_{\mathrm{f}}$ are the thrust, drag, and fuel mass flow, respectively.

At low altitudes and airspeeds, the equivalent airspeed, $V_{\mathrm{EAS}}$, can serve as a proxy for the indicated airspeed, and from the relationship with true airspeed, the following expression can be derived:

$$
V_{\mathrm{EAS}}=V_{\mathrm{TAS}} \sqrt{\rho / \rho_{0}}
$$

where $\rho$ is the ambient air density, and $\rho_{0}$ is the air density at sea level.

By combining Equations (1)-(4) with Equation (5), the equations of motion can be rewritten as follows:

$$
\begin{gathered}
\dot{V}_{\mathrm{EAS}}=\left\{g_{0}\left[\frac{(T-D)}{W}-\sin \gamma\right]+\frac{1}{2 \rho} \frac{\partial \rho}{\partial h} V_{\mathrm{TAS}}^{2} \sin \gamma\right\} \sqrt{\rho / \rho_{0}}, \\
\dot{s}=V_{\mathrm{EAS}} \sqrt{\rho / \rho_{0}} \cos \gamma, \\
\dot{h}=V_{\mathrm{EAS}} \sqrt{\rho / \rho_{0}} \sin \gamma, \\
\dot{W}=-\dot{m}_{\mathrm{f}} g_{0},
\end{gathered}
$$

where, $\frac{\partial \rho}{\partial h}$ is the derivative of the ambient air density with respect to altitude. 
With the use of Equations (6)-(9), the aircraft performance model has two control variables, viz. the flight path angle $\gamma$ and thrust $T$, and four state variables $x=\left[V_{\mathrm{EAS}}, h, s, W\right]$.

\subsection{The Trajectory Parameterization Technique}

As pointed out in Section 1, although the use of gradient-free optimization methods has many advantages, their computational cost is still quite large, notably spent on evaluating the objective function or constraints (or model evaluations). Therefore, one of the most efficient approaches to reduce the computational cost for these methods is to decrease the number of model evaluations as much as possible, especially by avoiding the evaluation of infeasible solutions during the optimization process. In trajectory optimization problems, operational constraints in different flight phases can cause the violation of new solutions found by an optimization algorithm, and hence there will be a large amount of computation time for evaluating these solutions while they are not potential candidates for an optimal solution. Recognizing this problem, Hartjes and Visser [19] introduced a novel trajectory parameterization that can handle operational constraints in the problem formulation, thus reducing the computational cost significantly. This technique aims to decompose a trajectory into a separate vertical path and ground track. The main advantage of this decomposition is that events in the vertical and horizontal plane can be decoupled, and that the overall number of optimization parameters can be reduced significantly without compromising the accuracy or degrees of freedom of the final solutions.

For ground track generation, a modern navigation technology known as required navigation performance (RNP) is applied. In RNP, track-to-a-fix (TF) and radius-to-a-fix (RF) leg types are often used to construct flight paths between waypoints. This is because of their ability to avoid noise-sensitive areas and minimizing flight track dispersion. By using these two segment types, the ground track can be generated using only straight legs and constant radius turns. An example of this is given in Figure 1, where the optimal design variables comprise of $L_{1}, L_{2}, R_{1}, R_{2}$, and $\Delta \chi_{1}$. When the initial and final position is known, the remaining parameters $L_{3}$ and $\Delta \chi_{2}$ can be determined analytically through a geometric relationship.

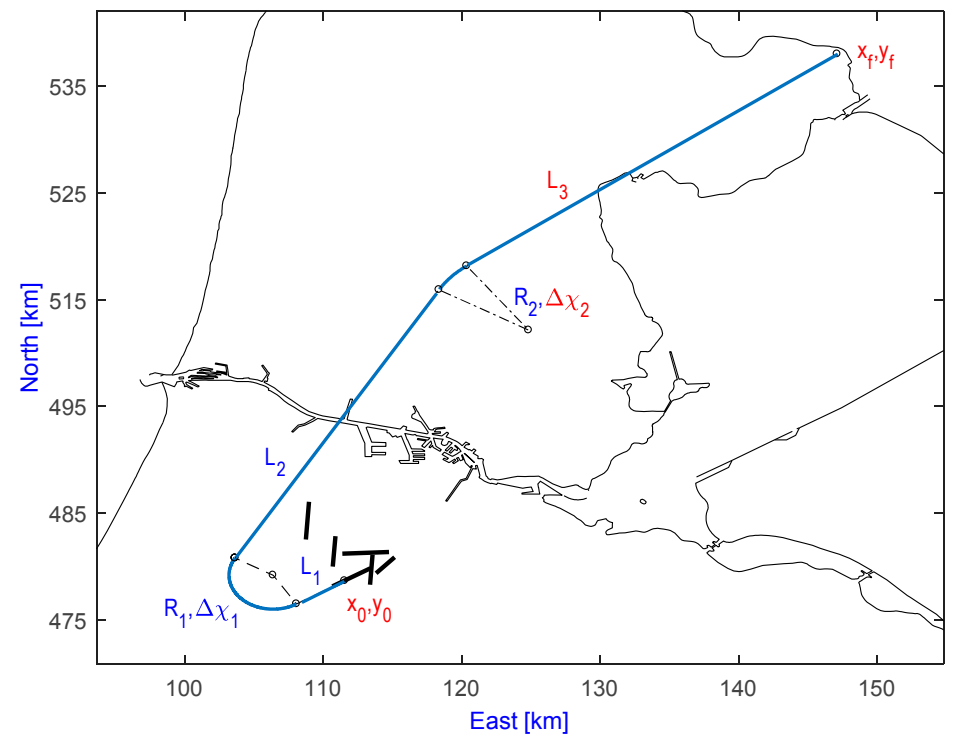

Figure 1. Ground track parameterization.

For the vertical path generation, the vertical profile is synthesized based on flight procedures derived from ICAO [26]. For instance, aircraft are not allowed to descend and/or decelerate during departure and ascend and/or accelerate during arrival. In order to parameterize this part, the trajectory is split into a number of segments. In each segment, two control inputs (i.e., flight path angle setting $\gamma_{n, i}$ and throttle setting $\Gamma_{n, i}$ ) are kept constant and are either directly assigned based on operational 
requirements or designated as optimal design variables. For each segment, the flight path angle $\gamma_{i}$ and thrust $T_{i}$ are set by adjusting their normalized control optimization parameters $\gamma_{n, i}\left(0 \leq \gamma_{n, i} \leq 1\right)$, and $\Gamma_{n, i}\left(0 \leq \Gamma_{n, i} \leq 1\right)$, respectively, as follows:

$$
\begin{aligned}
& \gamma_{i}=\left(\gamma_{\max , i}-\gamma_{\min , i}\right) \gamma_{n, i}+\gamma_{\min , i} \\
& T_{i}=\left(T_{\max , i}-T_{\min , i}\right) \Gamma_{n, i}+T_{\min , i}
\end{aligned}
$$

where the subscript $n$ presents a normalized control optimization parameter. The subscripts max, $i$ and min, $i$ indicate the maximum and minimum allowable values of the flight path angle and thrust for the $i$ th segment, which are specified based on the features of flight procedures.

In the departure procedure, $T_{\max }$ is set to be either maximum take-off thrust (TO) or maximum climb thrust (TCL) depending on the flight stage. In addition, because descending is not permitted in this phase $(\dot{h} \geq 0)$, the minimum flight path angle is set to zero $\left(\gamma_{\min }\right)$. In addition, with the assumption that $T_{\max }$ is selected and that the airspeed is maintained constant, $\gamma_{\max }$ can be determined from Equation (6). Finally, $T_{\min }$ can be determined as well from Equation (6), but now assuming level flight at constant speed is maintained. This yields the following equations:

$$
\begin{gathered}
\gamma_{\max }=\sin ^{-1}\left[\frac{-2 \rho g_{0} \cdot\left(T_{\max }-D\right)}{W\left(\frac{\partial \rho}{\partial h} V_{\mathrm{TAS}}^{2}-2 \rho g_{0}\right)}\right] \\
T_{\min }=D-\frac{W}{2 \rho g_{0}} \frac{\partial \rho}{\partial h} V_{\mathrm{TAS}}^{2} \sin \gamma_{\min }+W \sin \gamma_{\min } .
\end{gathered}
$$

In the arrival procedure, the process is reversed. The minimum thrust $T_{\min }$ is set equal to the idle thrust derived from the aircraft engine model, while the maximum flight path angle is set to be zero $\left(\gamma_{\max }=0\right)$, as ascending is not allowed in the approach. In addition, by assuming that the aircraft is not allowed to accelerate during descending, $T_{\max }$ can be determined by the same formula in Equation (13), replacing $T_{\min }$ by $T_{\max }$. The minimum flight path angle $\gamma_{\min }$ is evaluated with respect to the minimum thrust $T_{\min }$ by the same formula in Equation (12), replacing $T_{\max }$ and $\gamma_{\max }$ by $T_{\min }$ and $\gamma_{\min }$.

\section{Formulation of the Optimization Problem}

The main objective of the study is to design optimal departure routes which can help reduce considerably the adverse impact of aircraft noise on people living in the vicinity of airports. However, purely focusing on noise impact may result in a significant increase in fuel consumption, which is against the interests of stakeholders like airline companies. To balance this conflict, therefore, fuel consumption is also taken into account as the second objective.

While fuel consumption can readily be evaluated by the change of the aircraft weight from Equations (6)-(9), noise impact is harder to quantify. In order to measure the influence of aircraft noise on communities surrounding airports, the percentage of people who are likely to be awakened due to aircraft noise exposure is utilized in this paper. This criterion was proposed by the American National Standards Institute (ANSI) in 2008 and is defined as follows [27]:

$$
\% \text { Awakening }=\frac{1}{1+e^{-\left(-6.8884+0.04444 \mathrm{SEL}_{\text {indoor }}\right)}},
$$

where \%Awakening is the percentage of awakened people owing to the noise of an aircraft. $\mathrm{SEL}_{\text {indoor }}$ is the indoor sound exposure level in decibel $(\mathrm{dB})$ and is evaluated by using a replica of the integrated noise model (INM) that has been the Federal Aviation Authorities' (FAA) standard regulatory noise model since the late 1970s [19]. Because SEL obtained from INM represents an outdoor value, an amount of $15 \mathrm{~dB}$ is subtracted to obtain $\mathrm{SEL}_{\text {indoor }}$, accounting for the sound absorption of an average 
house with an open window [28]. It should be noted that only SEL values that are larger than or equal to $50 \mathrm{~dB}$ are taken into account, while those that are less than $50 \mathrm{~dB}$ are ignored, and their probabilities are set to zero [29].

By considering two objectives (noise and fuel) at the same time, a bi-objective optimization problem is formulated as follows:

$$
\begin{array}{cc}
\left.\min _{x, \gamma_{n}, \Gamma_{n}} \quad \text { \{number of awakenings, fuel burn }\right\} \\
\text { s.t. } & \mu \mu_{\max } \\
h_{\mathrm{f}} & =h_{\text {final }} \\
V_{\mathrm{EAS}, \mathrm{f}} & =V_{\mathrm{EAS}, \text { final }}
\end{array}
$$

where $x$ is the vector of ground track variables. $\gamma_{n}$ and $\Gamma_{n}$ are the vectors of the flight path angle setting and throttle setting variables for each segment. The variable $\mu$ is the bank angle, which is defined by $\mu= \pm \tan ^{-1}\left(\frac{V_{\text {TAS }}^{2}}{g_{0} R}\right)$. The parameter $\mu_{\max }$ is the allowable value of $\mu$, which is dependent on altitude as specified by ICAO [26]. The parameters $h_{\text {final }}$ and $V_{\text {EAS, final }}$ are, respectively, the prescribed final altitude and equivalent airspeed of the flight procedures.

\section{MOEA/D Algorithm and New Implementations}

\subsection{MOEA/D Algorithm}

The multi-objective evolutionary algorithm based on decomposition (MOEA/D), first proposed by Zhang and $\mathrm{Li}$ [21], has been recognized as one of the most popular multi-objective evolutionary algorithms to date [30]. In MOEA/D, a multi-objective optimization problem (MOP) is transformed into a set of single optimization sub-problems by applying decomposition approaches, and then evolutionary algorithms are utilized to optimize these sub-problems simultaneously. With the use of different decomposition methods and different evolutionary algorithms, various versions of MOEA/D have been developed in recent years, e.g., MOEA/D-DE [22], MOEA/D-DRA [31], MOEA/D-XBS [21], and MOEA/D-GR [23]. Although different variants of MOEA/D are available in literature, a powerful single version of MOEA/D that integrates different advantages of the current versions is not yet in place. With the aim of developing an efficient version of MOEA/D for real-life problems, a powerful MOEA/D version is therefore developed in this study that is a combination of MOEA/D-DE [22], an adaptive replacement strategy [23], a stopping condition criterion [24], and a constraint-handling technique [25]. The general framework of MOEA/D is presented in Algorithm 1. For more details, readers are encouraged to refer to [22-25,32].

\subsection{New Implementations}

Although MOEA/D has been demonstrated to be more efficient than NSGA-II and other methods, and in this study, its performance has also been strongly supported by the integration of the powerful features recently developed, like other population-based optimization methods, MOEA/D is still time-consuming, requiring a significant amount of model evaluations. Since the considered problem is a constrained optimization problem, there could be many trial solutions evaluated by MOEA/D during the optimization process that violate the constraints (or are infeasible). The evaluations of these solutions may lead to a significant increase in the computational cost of the algorithm, while they may not provide helpful information for searching an optimal solution. 


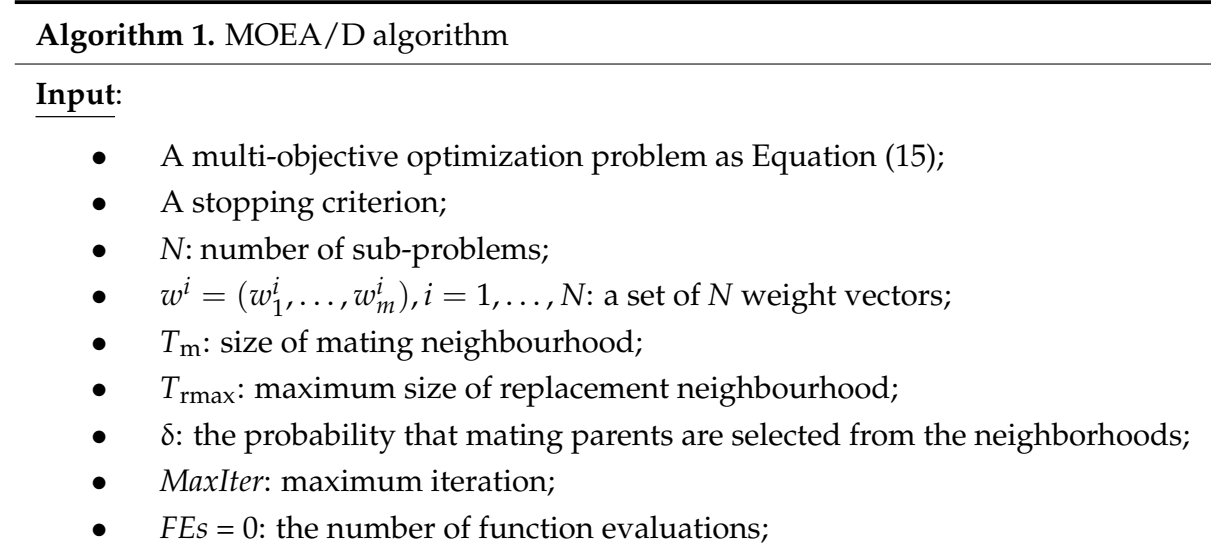

\section{Step 1. Initialization}

1.1. Find the $T_{\mathrm{m}}$ closest weight vectors to each weight vector based on the Euclidean distances of any two weight vectors. For each sub-problem $i=1, \ldots, N$ set $\boldsymbol{B}^{i}=\left(i_{1}, \ldots, i_{T_{\mathrm{m}}}\right)$ where $w^{i_{1}}, \ldots, w^{i_{T_{\mathrm{m}}}}$ are the closest weight vectors to $w^{i}$;

1.2. Create an initial population $\boldsymbol{P}=\left\{x^{i}, \ldots, x^{N}\right\}$ by uniformly randomly sampling from design space $\Omega$. Evaluate the fitness value $F V^{i}$ of each solution $x^{i}$, i.e., $F V^{i}=\left(f_{1}\left(x^{i}\right), \ldots, f_{m}\left(x^{i}\right)\right)$ and set $\boldsymbol{F V}=\left\{F V^{1}\left(\boldsymbol{x}^{1}\right), \ldots, F V^{N}\left(\boldsymbol{x}^{N}\right)\right\} ;$

1.3. Initialize ideal point $z^{*}=\left(z_{1}^{*}, \ldots, z_{m}^{*}\right)^{\mathrm{T}}$ by setting $z_{j}^{*}=\min \left\{f_{j}(x) \mid x \in \Omega, j=1, \ldots, m\right\}^{\mathrm{T}}$ and nadir point $z^{\text {nad }}=\left(z_{1}^{\text {nad }}, \ldots, z_{m}^{\text {nad }}\right)^{\mathrm{T}}$ by setting $z_{j}^{*}=\max \left\{f_{j}(x) \mid x \in \Omega, j=1, \ldots, m\right\}^{\mathrm{T}} ;$

1.4. Set $F E s=F E s+N$, and generation: $g e n=1$;

\section{Step 2. Update}

while (the stopping condition is not satisfied)

for $i=1, \ldots, N$; do

2.1. Selection of mating/update range

$$
\text { Set } \boldsymbol{B}_{m}\left\{\begin{array}{cc}
\boldsymbol{B}^{i} & \text { if rand }<\delta \\
\{1, \ldots, N\} & \text { otherwise }
\end{array}\right.
$$

where rand is a uniformly distributed random number in [0, 1];

2.2. Reproduction: randomly select three parent individuals $r_{1}, r_{2}$ and $r_{3}\left(r_{1} \neq r_{2} \neq r_{3} \neq i\right)$ from $\boldsymbol{B}_{m}$ and generate a solution $\bar{y}$ by applying "DE/rand/1" operator, and then perform a mutation operator on $\bar{y}$ to create a new solution $y$;

2.3. Repair: if any element of $y$ is out of $\Omega$, its value will be randomly regenerated inside $\Omega$;

2.4. Evaluate the fitness value of new solution $y$;

2.5. Update of $z^{*}$ and $z^{\text {nad: }}$ for each $j=1, \ldots, m$ if $z_{j}^{*} \leq f_{j}\left(x^{i}\right)$ then set $z_{j}^{*}=f_{j}\left(x^{i}\right)$, and if $z_{j}^{\text {nad }} \geq f_{j}\left(x^{i}\right)$ then set $z_{j}^{\text {nad }}=f_{j}\left(x^{i}\right)$;

2.6. Update of solutions: use an adaptive replacement strategy in [23]:

\section{end for}

Set $F E s=F E s+N$, and $g e n=g e n+1$;

\section{Step 3. Stopping condition}

Use a stopping criterion in [24].

if (stopping criterion is satisfied or MaxIter is reached)

Stop the algorithm;

end if

end while

Output: Pareto set PS $=\left\{x^{1}, \ldots, x^{N}\right\} ;$ Pareto front $\mathbf{P F}=\left\{F V^{1}\left(x^{1}\right), \ldots, F V^{1}\left(x^{N}\right)\right\}$. 
In an effort to avoid the above problem, a new implementation on the setting of the optimization problem and MOEA/D is introduced. More specifically, for the considered problem, the computational cost is mainly spent on two main tasks: one for solving the ODEs to obtain the fuel objective and the necessary inputs for calculating noise and to evaluate the constraints of the optimization problem (for example, the final conditions of velocity and altitude, and the bank angle constraints), and the other for computing the noise objective. While the computational cost of the first task is not so significant, the computational expense of the second task is quite considerable. In MOEA/D, after a new solution is found, its objectives and constraints are measured and compared with those of previous solutions. The solutions with the better objectives and/or the better level of constraint violation will be selected for the next generation. It is readily clear that if there is a comparison between a feasible solution and an infeasible solution, the feasible one will be selected, and in the case of comparing two infeasible solutions, the solution with the lowest level of constraint violation will be chosen. By recognizing this feature, a new decision has been made for the algorithm to decide whether or not the noise calculation of a new solution is executed. Particularly, after the fuel objective and constraints of a new solution are assessed, a quick check and comparison of the level of constraint violation between them are executed first. If the new solution violates the constraints or has a higher level of constraint violation compared to, respectively, a feasible solution or an infeasible solution at the previous generation, its noise calculation will not be executed. In that case, the update procedures 2.5 and 2.6 of MOEA/D in Algorithm 1 are ignored as well.

With the above new implementation, the computational cost of noise calculation only is spent on either feasible solutions or on the infeasible solution with the lowest level of constraint violation, and hence the computational cost of the whole optimization process will be significantly reduced.

\section{Numerical Example}

In order to demonstrate the capabilities and efficiencies of MOEA/D for conducting the optimal design of noise abatement departure routes, two standard instrument departures (SID) currently in use at Schiphol Airport are considered in this section. The first SID is called SPIJKERBOOR2K, which starts at runway 24 and finishes at the ANDIK intersection, and the other one is ARNEM2N, which starts at runway 09 and terminates at the IVLUT intersection, as shown in Figure 2. These routes pass closely by the communities of Hoofddorp, Haarlem, and Amstelveen, where most of the noise nuisance occurs. For both departures, the optimized trajectory starts at $35 \mathrm{ft}$, a take-off safety speed of $V_{2}+10 \mathrm{kts}$, with the landing gear retracted and departure flaps selected and is terminated at an altitude of $6000 \mathrm{ft}$ and an equivalent airspeed (EAS) of $250 \mathrm{kts}$. The ground tracks are constructed by three straight legs and two turns, as shown in Figure 2, which results in five design variables, while the vertical path is subdivided into 10 segments and parameterized as the study in [19], which results in an additional 18 design variables. For the reference case, the ground track is fixed to conform to the current SID, while the vertical path is optimized for fuel burn after finishing the NADP-1. The details of the parameterization can be found in Hartjes and Visser [19]. Two noise-exposed regions of $66 \mathrm{~km} \times 59 \mathrm{~km}$ and $36.5 \mathrm{~km} \times 20 \mathrm{~km}$ with a population grid cell size of $500 \mathrm{~m} \times 500 \mathrm{~m}$ [33] are used for the SPIJKERBOOR2K and ARNEM2N SIDs, respectively. A Boeing 737-800 with two engines is used as the aircraft model, based on the Base of Aircraft Data (BADA), with an initial mass of 68 tons ( $85 \%$ of the maximum take-off weight) as a representative take-off mass.

To compare the performance of MOEA/D, the well-known NSGA-II [34] is also applied to solve these problems. A population size of 50 is used for both methods, and the algorithms will stop when either their convergence criteria are satisfied or the maximum number of iterations (MaxIter) is reached, where MaxIter is set at 1000. All algorithms are implemented in Matlab 2016b on a Core i5, 8 GB RAM desktop. 


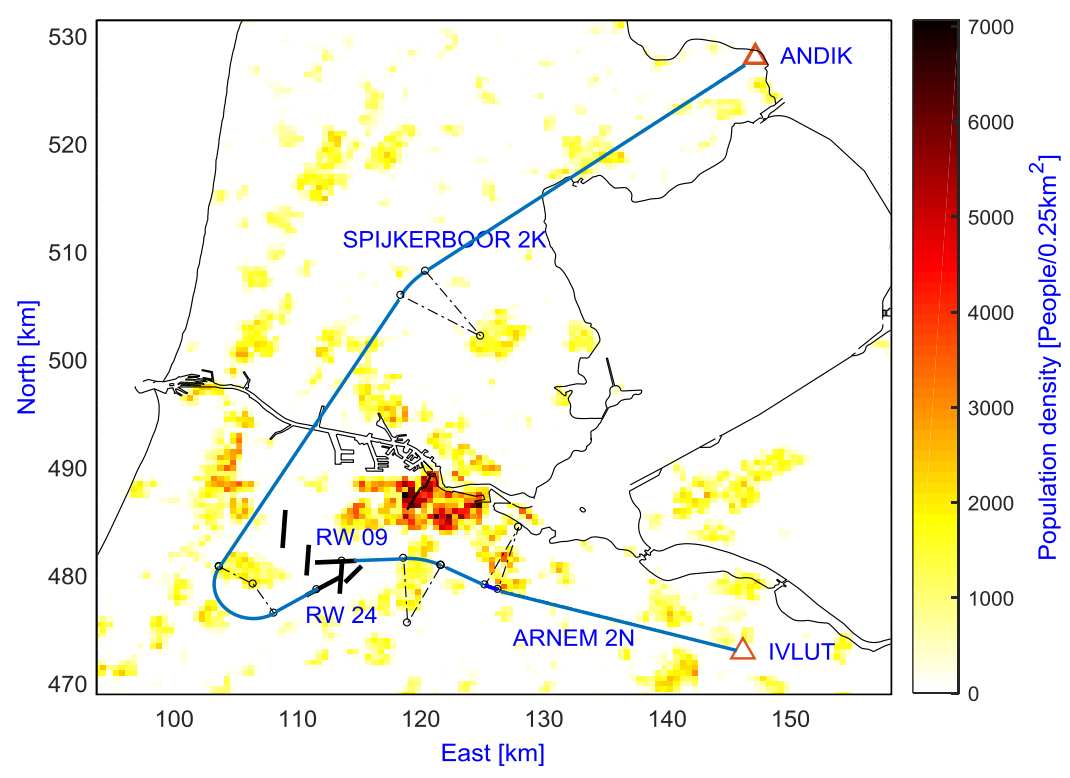

Figure 2. Departure scenarios.

\subsection{Departure Route SPIJKERBOOR2K}

The Pareto-optimal solutions obtained by the methods are shown in Figure 3, and their corresponding ground tracks are illustrated in Figure 4. The cases highlighted in Figure 3 by a yellow star and red circle are also highlighted in Figure 4 and further discussed in the text. From a comparison of solution methods in Figure 3, it can be seen that the quality of solutions obtained by MOEA/D is generally better than those achieved by NSGA-II. Specifically, MOEA/D provides many solutions that dominate those of NSGA-II with a significantly lower computational effort. In order to achieve these results, MOEA/D requires 23,357 model evaluations, in which only 8399 involve a noise calculation. Hence, the total computation time is only $2.2 \mathrm{~h}$, while NSGA-II requires 50,500 evaluations resulting in $9.59 \mathrm{~h}$ computation time for a full evaluation.

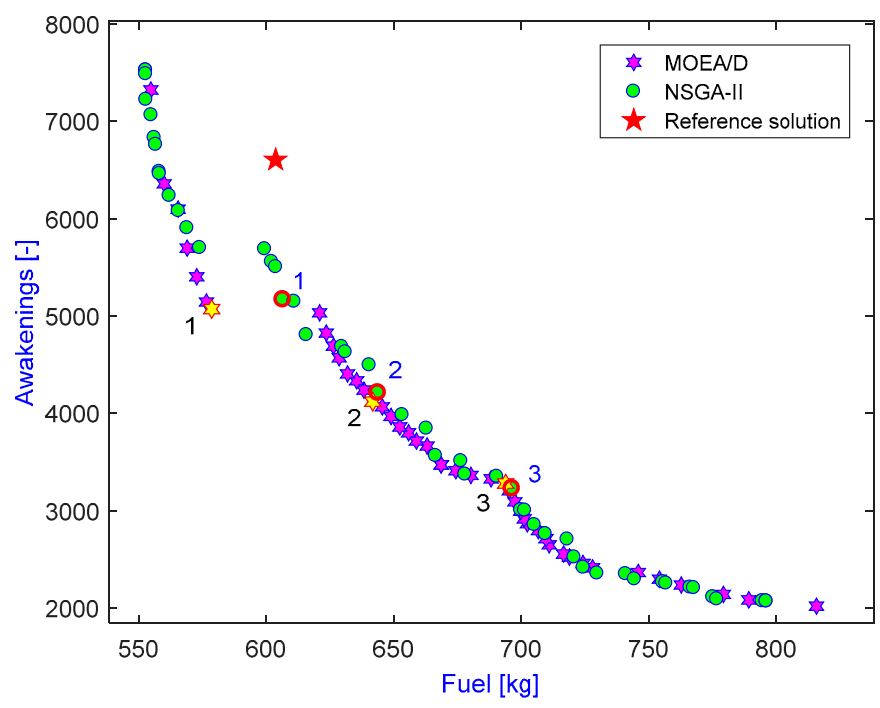

Figure 3. Comparison of optimal solutions obtained by NSGA-II, MOEA/D, and the reference case.

From an engineering point of view, it can be seen that the obtained ground tracks in Figure 4 are quite reasonable and appropriate. There are three different groups of ground tracks generated by MOEA/D and four groups by NSGA-II, all of them trying to avoid noise-sensitive communities. 
These results also help to explain why there are some gaps in the Pareto fronts. In comparison to the reference case, it can be observed that most of the optimal solutions offer a better environmental performance. In particular, as shown in the left of Figure 3, there are some cases that perform better for both objectives, whereas the remaining solutions on the right are much better regarding awakenings, although there is a slight increase in fuel burn.
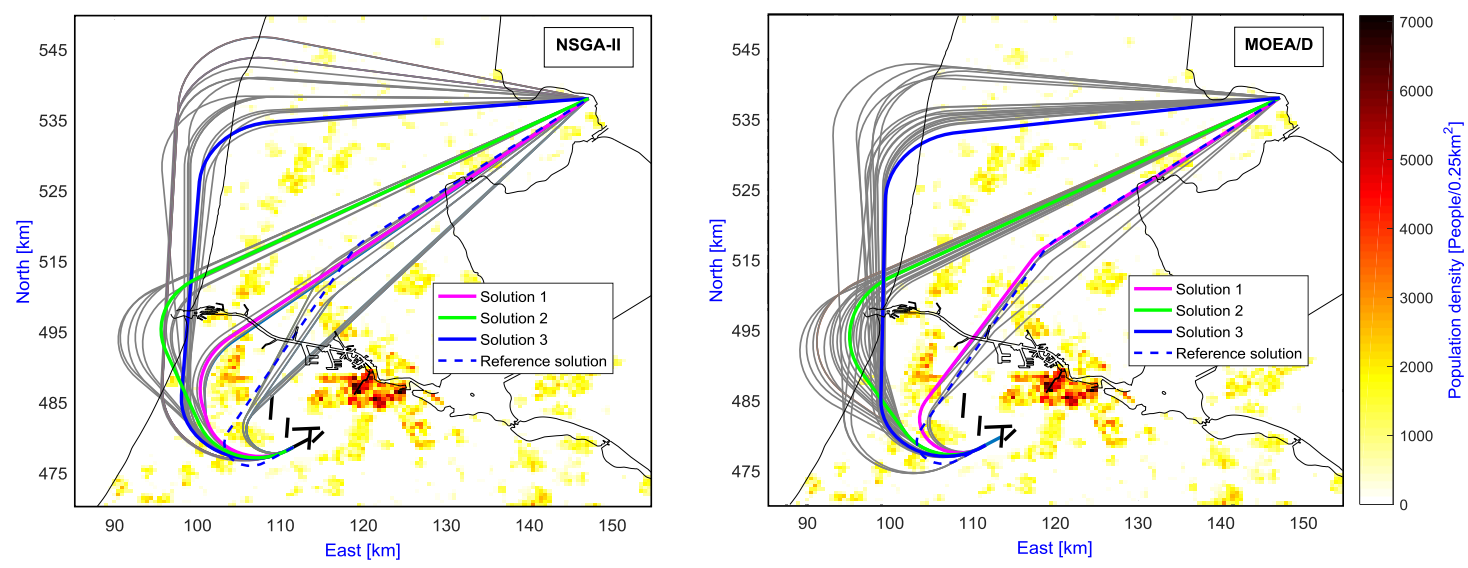

Figure 4. Optimal ground tracks obtained by NSGA-II (left) and MOEA/D (right).

For a performance comparison, the number of people expected to be awakened and the fuel burn of the three representative cases are extracted from the Pareto fronts (numbered as shown in Figures 3 and 4) and listed in Table 1 along with the results of the reference case. The vertical profiles of the MOEA/D computed cases are given in Figure 5. Compared to the reference case, it can be seen that all solutions are non-dominated, either better at awakenings and worse at fuel burn or vice versa, except for case 1. Despite having almost the same ground track length with an optimal combination of the ground track and vertical profile, case 1 offers much better performance in terms of all three criteria. From Table 1, it can also be seen that the difference between the objective values of the three representative cases is significant, especially in terms of the number of awakenings. A relatively small detour allows a significant part of the communities surrounding the airport to be avoided, and, considering the exponential relationship between noise and awakenings, consequently leads to a significant reduction in the noise criterion. By taking a closer look at Figure 5, it can be seen that in the first phase of flight, for all optimal cases, the aircraft prefers to fly at a low altitude with a high speed to pass over populated regions. This is because the spread of aircraft noise at a low altitude is smaller than that at a higher altitude due to increased lateral attenuation, and hence it leads to a significant reduction of awakenings. Also, maintaining a low altitude allows the aircraft to accelerate to a high airspeed sooner, which leads to lower exposure times and hence to lower SEL-values, while the source noise levels do not increase significantly. From Figure 5, it also becomes clear that the time during which the aircraft stays at a low altitude is dependent on the population density distribution underneath the flight path. This is also clearly depicted in Figure 6, where the noise level contours of cases 2 and 3 are illustrated. 
Table 1. Comparison of objectives of cases 1-3 and the reference case.

\begin{tabular}{ccccc}
\hline \multicolumn{2}{c}{ Case Number } & Time (s) & Fuel (kg) & Awakening \\
\hline \multirow{2}{*}{1} & MOEA/D & 569.69 & 578.66 & 5072 \\
& NSGA-II & 612.68 & 607.91 & 5199 \\
\hline \multirow{2}{*}{2} & MOEA/D & 666.38 & 641.81 & 4121 \\
& NSGA-II & 668.63 & 643.76 & 4213 \\
\hline \multirow{2}{*}{3} & MOEA/D & 736.27 & 693.90 & 3280 \\
& NSGA-II & 747.17 & 696.35 & 3232 \\
\hline \multicolumn{2}{r}{ Reference solution } & 595.11 & 603.69 & 6602 \\
\hline
\end{tabular}
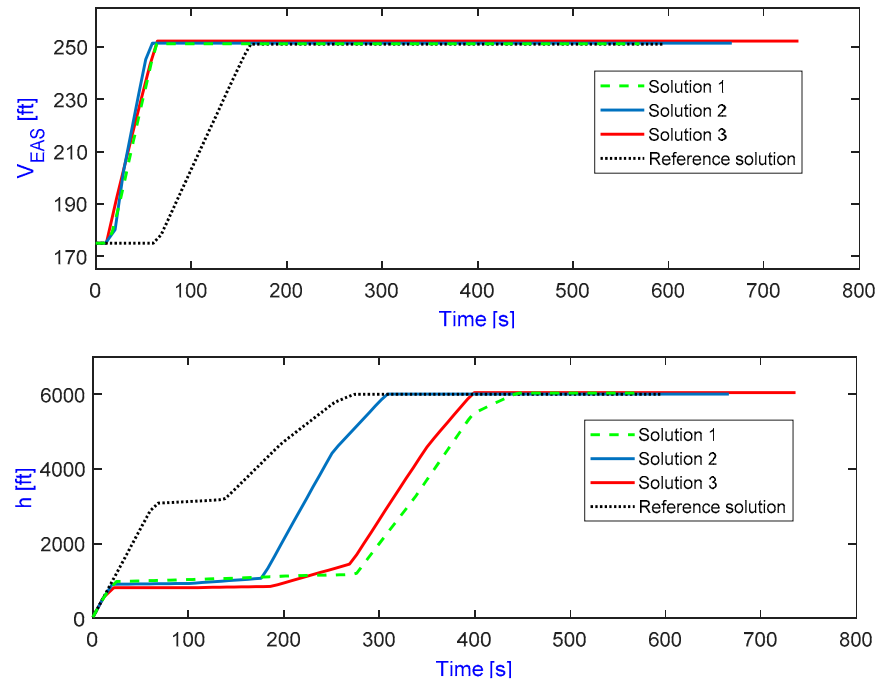

Figure 5. Vertical profiles of cases 1-3, and the reference case.
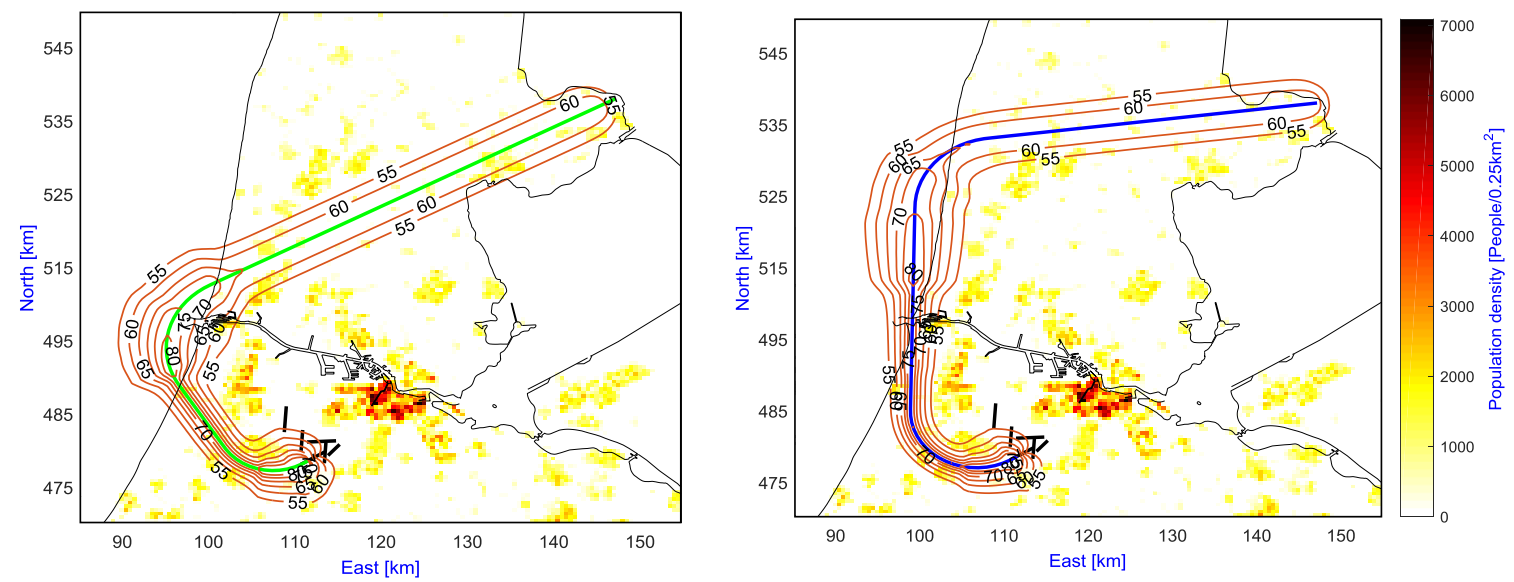

Figure 6. Noise level contours for cases 2 (left) and 3 (right).

\subsection{Departure Route ARNEM2N}

The Pareto-optimal solutions obtained by MOEA/D and NSGA-II for this example are illustrated in Figure 7, while the ground tracks are provided in Figure 8. Again, some representative cases are highlighted in the figures. To acquire these results, NSGA-II requires 45,000 model evaluations in $4.36 \mathrm{~h}$, while MOEA/D converges after 24,122 model evaluations in $1.99 \mathrm{~h}$, with only 17,010 requiring a noise calculation. Compared to the previous example, the obtained ground tracks, in this case, are more divergent. This is because there is no area with a high concentration of population within the 
investigated region except for a small area close to runway 09. However, this region has been avoided by most of the optimal solutions. It can also be seen from Figure 7 that all optimal solutions dominate the reference case.

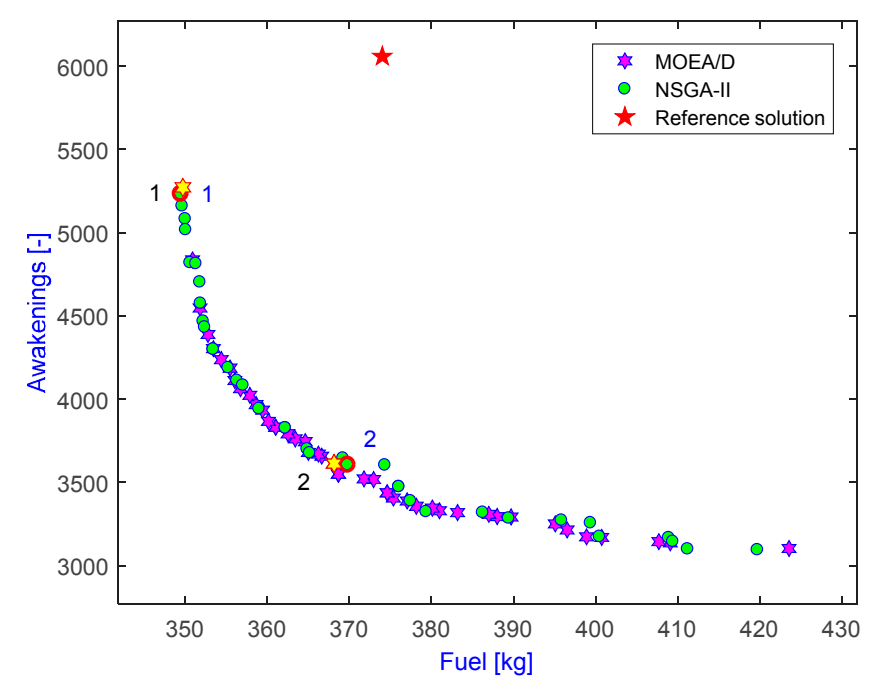

Figure 7. Comparison of optimal solutions obtained by NSGA-II and MOEA/D and the reference case.
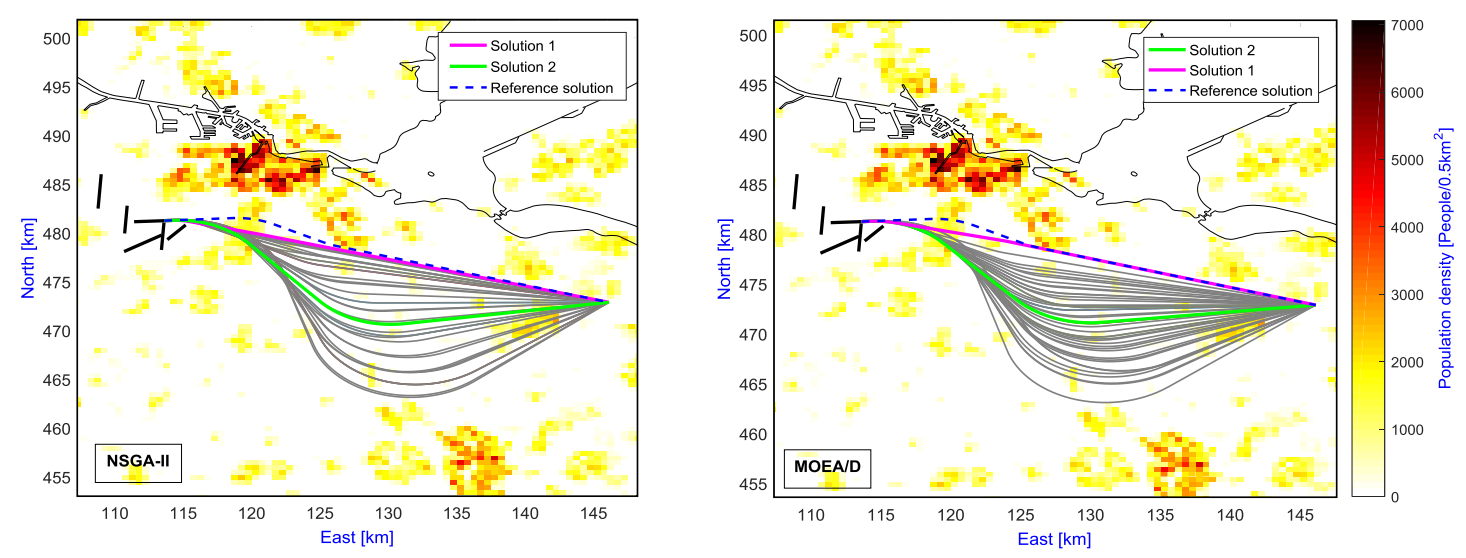

Figure 8. Optimal ground tracks obtained by NSGA-II (left) and MOEA/D (right).

For a comparison of specific values, the number of awakenings and fuel burn of the representative cases extracted from the Pareto-optimal solutions (as highlighted in Figure 7) are given in Table 2. The vertical profiles of the MOEA/D cases are also provided in Figure 9. From the table, it can be seen that all optimal cases have better results regarding fuel burn and awakenings compared to the reference case, while case 1 significantly outperforms the reference case on all considered criteria (i.e., fuel burn, awakenings, and time). As the airspeed and altitude histories are concerned, their behavior is almost the same as in the previous example. The aircraft often fly at a low attitude with a relatively high speed when passing over densely populated areas in the first phases, while the time during which an aircraft flies at a low attitude depends on the population distribution underneath a flight path.

Summarizing, based on the obtained results, it can be concluded that MOEA/D is an effective method for solving the optimal design problem of noise abatement departure routes. Compared to NSGA-II, MOEA/D generally outperforms NSGA-II in terms of both the quality of solutions and computation time. 
Table 2. Comparison of objectives of cases 1-2 and the reference case.

\begin{tabular}{ccccc}
\hline \multicolumn{2}{c}{ Case Number } & Time (s) & Fuel (kg) & Awakening \\
\hline \multirow{2}{*}{1} & MOEA/D & 257.51 & 349.71 & 5271 \\
& NSGA-II & 256.29 & 349.46 & 5232 \\
\hline \multirow{2}{*}{2} & MOEA/D & 278.81 & 368.14 & 3613 \\
& NSGA-II & 281.19 & 370.28 & 3634 \\
\hline \multicolumn{2}{l}{ Reference solution } & 271.14 & 374.01 & 6058 \\
\hline
\end{tabular}
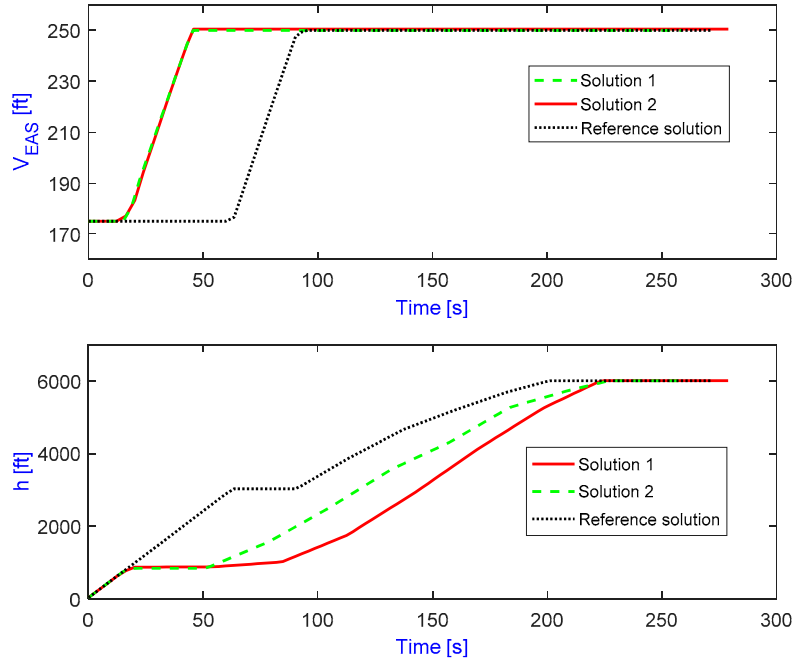

Figure 9. Vertical profiles of cases 1-2, and the reference solution.

\section{Conclusions}

In this study, a novel and efficient application of MOEA/D for the optimal design of noise abatement departure routes is presented. Besides the typical advantages, the performance of MOEA/D is also considerably enhanced by the integration of recently developed features, which include an adaptive replacement strategy, a stopping condition criterion, and a constraint-handling technique. Also, the performance of the entire optimization process is significantly improved by the implementation on the setting of the optimization problem and the MOEA/D algorithm. Owing to this implementation, the computational cost of solving the optimization problems is sharply reduced.

The applicability and effectiveness of MOEA/D and the new implementations are demonstrated through two example scenarios of departure routes at Schiphol Airport in the Netherlands: SPIJKERBOOR2K and ARNEM2N. For comparison purposes, NSGA-II is also applied to solve these problems. The comparative results show that MOEA/D is generally better than NSGA-II when considering the quality of solutions and much better regarding the convergence rate and overall computational cost.

With these promising results, in future work, MOEA/D will be extended to consider different routes at other airports, and its performance will also be investigated in different associated problems like route and runway allocations. Furthermore, the performance of the algorithm will also be further enhanced to deal with large and complex problems, especially in the distribution of solutions and the convergence rate.

Author Contributions: Vinh Ho-Huu developed the optimization algorithm, performed the numerical examples, and wrote the manuscript. Sander Hartjes proposed the idea and analyzed the results. Hendrikus G. Visser and Richard Curran provided important feedback on the proposed approach and contributed to the review of the paper.

Conflicts of Interest: The authors declare no conflict of interest. 


\section{References}

1. Boeing. Current Market Outlook 2016-2035. Available online: http://www.boeing.com/resources/ boeingdotcom/commercial/about-our-market/assets/downloads/cmo_print_2016_final_updated.pdf (accessed on 12 July 2017).

2. Hartjes, S.; Dons, J.; Visser, H.G. Optimization of Area Navigation Arrival Routes for Cumulative Noise Exposure. J. Aircr. 2014, 51, 1432-1438. [CrossRef]

3. Clean Sky. Available online: http:/ / www.cleansky.eu/\#no-back (accessed on 23 October 2017).

4. Atlantic Interoperability Initiative to Reduce Emissions (AIRE). Available online: https:/ / ec.europa.eu/ transport/modes/air/environment/aire_en (accessed on 23 October 2017).

5. Asia and South Pacific Initiative to Reduce Emissions (ASPIRE). Available online: http:/ /www.aspire-green. $\mathrm{com} /$ (accessed on 23 October 2017).

6. Marais, K.B.; Reynolds, T.G.; Uday, P.; Muller, D.; Lovegren, J.; Dumont, J.-M.; Hansman, R.J. Evaluation of potential near-term operational changes to mitigate environmental impacts of aviation. Proc. Inst. Mech. Eng. Part G J. Aerosp. Eng. 2013, 227, 1277-1299. [CrossRef]

7. Visser, H.G. Generic and site-specific criteria in the optimization of noise abatement tarajectories. Transp. Res. Part D Transp. Environ. 2005, 10, 405-419. [CrossRef]

8. Green, J.E. Air Travel-Greener by Design Mitigating the environmental impact of aviation: Opportunities and priorities. Aeronaut. J. 2005, 109, 361-416.

9. Visser, H.G.; Wijnen, R.A.A. Optimization of Noise Abatement Departure Trajectories. J. Aircr. 2001, 38, 620-627. [CrossRef]

10. Visser, H.G.; Wijnen, R.A.A. Optimisation of noise abatement arrival trajectories. Aeronaut. J. 2003, 107, 607-615.

11. Hartjes, S.; Visser, H.G.; Hebly, S.J. Optimisation of RNAV noise and emission abatement standard instrument departures. Aeronaut. J. 2010, 114, 757-767. [CrossRef]

12. Hogenhuis, R.H.; Hebly, S.J.; Visser, H.G. Optimization of area navigation noise abatement approach trajectories. Proc. Inst. Mech. Eng. Part G J. Aerosp. Eng. 2011, 225, 513-521. [CrossRef]

13. Braakenburg, M.L.; Hartjes, S.; Visser, H.G. Development of a Multi-Event Trajectory Optimization Tool for Noise-Optimized Approach Route Design. AIAA 2011, 1-13. [CrossRef]

14. Prats, X.; Puig, V.; Quevedo, J.; Nejjari, F. Lexicographic optimisation for optimal departure aircraft trajectories. Aerosp. Sci. Technol. 2010, 14, 26-37. [CrossRef]

15. Prats, X.; Puig, V.; Quevedo, J.; Nejjari, F. Multi-objective optimisation for aircraft departure trajectories minimising noise annoyance. Transp. Res. Part C Emerg. Technol. 2010, 18, 975-989. [CrossRef]

16. Khardi, S.; Abdallah, L. Optimization approaches of aircraft flight path reducing noise: Comparison of modeling methods. Appl. Acoust. 2012, 73, 291-301. [CrossRef]

17. Matthes, S.; Grewe, V.; Dahlmann, K.; Frömming, C.; Irvine, E.; Lim, L.; Linke, F.; Lührs, B.; Owen, B.; Shine, K.; et al. A Concept for Multi-Criteria Environmental Assessment of Aircraft Trajectories. Aerospace 2017, 4, 42. [CrossRef]

18. Torres, R.; Chaptal, J.; Bès, C.; Hiriart-Urruty, J.-B. Optimal, Environmentally Friendly Departure Procedures for Civil Aircraft. J. Aircr. 2011, 48, 11-23. [CrossRef]

19. Hartjes, S.; Visser, H. Efficient trajectory parameterization for environmental optimization of departure flight paths using a genetic algorithm. Proc. Inst. Mech. Eng. Part G J. Aerosp. Eng. 2016, 231, 1115-1123. [CrossRef]

20. Zhang, M.; Filippone, A.; Bojdo, N. Multi-objective departure trajectory optimisation of commercial aircraft on environmental impacts. In Proceedings of the Greener Aviation Conference, Brussels, Belgium, 11-13 October 2016.

21. Zhang, Q.; Li, H. MOEA/D: A Multiobjective Evolutionary Algorithm Based on Decomposition. IEEE Trans. Evol. Comput. 2007, 11, 712-731. [CrossRef]

22. Li, H.; Zhang, Q. Multiobjective Optimization Problems with Complicated Pareto Sets, MOEA/D and NSGA-II. IEEE Trans. Evol. Comput. 2009, 13, 284-302. [CrossRef]

23. Wang, Z.; Zhang, Q.; Zhou, A.; Gong, M.; Jiao, L. Adaptive Replacement Strategies for MOEA/D. IEEE Trans. Cybern. 2016, 46, 474-486. [CrossRef] [PubMed]

24. Abdul Kadhar, K.M.; Baskar, S. A stopping criterion for decomposition-based multi-objective evolutionary algorithms. Soft Comput. 2016, 1-20. [CrossRef] 
25. Jan, M.A.; Khanum, R.A. A study of two penalty-parameterless constraint handling techniques in the framework of MOEA/D. Appl. Soft Comput. J. 2013, 13, 128-148. [CrossRef]

26. International Civil Aviation Organization. Procedures for Air Navigation Services-Aircraft Operations; Flight Procedures, ICAO Document Number 8168; International Civil Aviation Organization: Montreal, QC, Canada, 2006; Volume I.

27. ANSI/ASA. Quantities and Procedures for Description and Measurement of Environmental Sound_Part 6: Methods for Estimation of Awakenings Associated with Outdoor Noise Events Heard in Homes; ANSI/ASA: Washington, DC, USA, 2008.

28. Miller, N.P.; Schomer, P.D. How many people will be awakened by noise tonight? Acoust. Today 2009, 5, 26-31. [CrossRef]

29. Yu, H.; Van Kampen, E.-J.; Mulder, J.A. An Optimization Paradigm for Arrival Trajectories usingTrajectory Segmentation and State Parameterization. AIAA Guid. Navig. Control Conf. 2016, 1-17. [CrossRef]

30. Trivedi, A.; Srinivasan, D.; Sanyal, K.; Ghosh, A. A Survey of Multiobjective Evolutionary Algorithms based on Decomposition. IEEE Trans. Evol. Comput. 2016, 440-462. [CrossRef]

31. Zhang, Q.; Liu, W.; Li, H. The performance of a new version of MOEA/D on CEC09 unconstrained MOP test instances. IEEE Congr. Evol. Comput. 2009, 203-208.

32. Ho-Huu, V.; Hartjes, S.; Visser, H.G.; Curran, R. An improved MOEA/D algorithm for bi-objective optimization problems with complex Pareto fronts and its application to structural optimization. Expert Syst. Appl. 2017. [CrossRef]

33. Xue, M.; Zelinski, S. Integrated Arrival- and Departure-Schedule Optimization Under Uncertainty. J. Aircr. 2015, 52, 1437-1443. [CrossRef]

34. Deb, K.; Pratab, S.; Agarwal, S.; Meyarivan, T. A Fast and Elitist Multiobjective Genetic Algorithm: NSGA-II. IEEE Trans. Evol. Comput. 2002, 6, 182-197. [CrossRef]

(C) 2017 by the authors. Licensee MDPI, Basel, Switzerland. This article is an open access article distributed under the terms and conditions of the Creative Commons Attribution (CC BY) license (http:/ / creativecommons.org/licenses/by/4.0/). 